\title{
Synthesis and Crystal Structure of Cerium(III) Picolinate Complex
}

\author{
Yunghee Oh, Jang Yong Kim, Hye Jin Kim, Taewoo Lee, ${ }^{\dagger}$ and Sung Kwon Kang ${ }^{\dagger, *}$ \\ Department of Chemistry, Dongeui University, Pusan 614-714, Korea \\ †Department of Chemistry, Chungnam National University, Daejeon 305-764, Korea. *E-mail: skkang@cnu.ac.kr \\ Received January 15, 2010, Accepted February 2, 2010
}

Key Words: Cerium, Picolinate, Pyridoin, X-ray crystal structure, Lanthanide

Lanthanide complexes containing organic ligand with conjugate group have been widely studied as those show characteristic luminescent properties, which are applied to biomedical, sensing and industrial fields. ${ }^{1}$ The luminescent properties originating from $4 \mathrm{f}-4 \mathrm{f}, 5 \mathrm{~d}-4 \mathrm{f}$ and charge transfer transition of lanthanide ions can be controlled to construct artificially molecular design. ${ }^{2}$ However, the design and preparation of lanthanide complexes is more difficult than that of transition metal complexes because of high coordination number of lanthanide ions. Therefore, the coordination chemistry as well as luminescence of lanthanides has been another challenging research field to the synthetic scientists in recent decades.

Pyridine carboxylates such as picolinate and nicotinate have been widely used to build lanthanide complexes because those molecules with nitrogen and oxygen atoms could efficiently transfer energy to lanthanide ion. ${ }^{4-5} \mathrm{We}$ are interested to make lanthanide complexes containing picolinate (pic) using $\alpha$-pyridoin instead of picolinic acid, since the reaction with $\alpha$-pyridoin is more clear and the yield is higher than using picolinic acid. We have already reported the preparation and crystal structures of europium and terbium picolinate complexes, $\mathrm{Na}\left[\mathrm{Ln}(\text { pic })_{4}\right]$. $2 \mathrm{H}_{2} \mathrm{O}(\mathrm{Ln}=\mathrm{Eu}(\mathrm{III}), \mathrm{Tb}(\mathrm{III}))$ using $\alpha$-pyridoin. ${ }^{6}$ This work is a continuation of preparation and structural characterization studies of lanthanide complexes.

Crystal structures of lanthanide picolinates have been reported as polymeric or mononuclearic structure. Most of them contained eight to nine coordinated metal with tris or tetrakis picolinates and water molecules, for example, $\left[\mathrm{Ln}(\mathrm{pic})_{3}\right] \cdot \mathrm{nH}_{2} \mathrm{O}$ $\left(\mathrm{Ln}=\mathrm{Sm},{ }^{7} \mathrm{Nd}^{7}{ }^{\mathrm{Dy}} \mathrm{8}^{8}\right)$ or $\mathrm{M}\left[\mathrm{Ln}(\mathrm{pic})_{4}\right] \cdot \mathrm{nH}_{2} \mathrm{O}(\mathrm{M}=$ counter cation, $\left.\mathrm{Ln}=\mathrm{Eu},{ }^{6} \mathrm{~Tb}^{6}{ }^{6} \mathrm{Nd},{ }^{9-10} \mathrm{Ho},{ }^{11} \mathrm{Er}^{12}{ }^{\mathrm{Sm}^{13}}\right)$. We have successfully prepared new cerium(III) picolinate complex, 1, from the reaction of $\mathrm{CeCl}_{3} \cdot 7 \mathrm{H}_{2} \mathrm{O}$ and $\alpha$-pyridoin anion in methanol and characterized by X-ray crystallography, leading to a ten-coordinated cerium compound containing five picolinate ligands.

\section{Experimental Section}

Synthesis and crystal growth. The $\alpha$-pyridoin $(0.630 \mathrm{~g}, 3$ mmol) was dissolved in $20 \mathrm{~mL}$ methanol and $3 \mathrm{~mL}$ of $1 \mathrm{M} \mathrm{NaOH}$ aqueous solution was added into the solution with stirring to generate $\alpha$-pyridoin anion. The solution color turned to yellow. Cerium chloride hepta hydrate $(0.372 \mathrm{~g}, 1 \mathrm{mmol})$ was dissolved in $10 \mathrm{~mL}$ methanol and added into the $\alpha$-pyridoin anionic solution. The reaction mixture was stirred for one hour and kept at room temperature overnight. Solids were filtered off and the filtrate was kept in refrigerator for three weeks. Crystals were filtered, washed by methanol and dried under vacuum. Yield was $82 \%$ and the product was thermally decomposed at $390{ }^{\circ} \mathrm{C}$. The reaction is summarized in Scheme 1.

Determination and refinement of the $\mathbf{X}$-ray Structure. The data for X-ray structure determination was collected on a Bruker SMART APEX-II CCD diffractormeter equipped with graphite monochromated Mo K $\alpha$ radiation $(\lambda=0.71073 \AA)$ at $295 \mathrm{~K}$. Structure was solved by applying the direct method using a SHELXS97 and refined by a full-matrix least-squares calculation on $F^{2}$ using SHELXL97. ${ }^{14}$ All non-hydrogen atoms were placed in ideal positions and were riding on their respective carbon atoms $\left(B_{\text {iso }}=1.2 B_{\text {eq }}\right)$. Crystallographic data for the structure reported here have been deposited with the Cambridge Crystallographic Data Centre (Deposition No. CCDC 761591). The data can be obtained free of charge via http://www.ccdc. cam.ac.uk/perl/catreq.cgi (or from the CCDC, 12 Union Road, Cambridge CB2 1EZ, UK; Fax: +44 1223 336033; e-mail: deposit@ccdc.cam.ac.uk).

\section{Results and Discussion}

The complex crystallizes in the monoclinic space $P 2_{1} / n$ as given in Table 1 for the crystallographic data and structure refinement parameters of complex $\mathbf{1}, \mathrm{Na}_{2}\left[\mathrm{Ce}(\text { pic })_{5}\right] \cdot \mathrm{picH} \cdot 4 \mathrm{H}_{2} \mathrm{O}$.

An ORTEP diagram of including the atom numbering scheme is shown in Figure 1. The structural feature of complex $\mathbf{1}$ is that five picolinate (pic) ligands are coordinated directly to the $\mathrm{Ce}$ atom in the inner coordination sphere forming the complex anion, while two $\mathrm{Na}$ atoms as counter cations are bound to the complex anion. In addition, there are one picolinic acid (picH) and four water molecules in the outer sphere.

$$
\alpha \text {-pyridoin/ } \mathrm{NaOH}+\mathrm{CeCl}_{3} \cdot 7 \mathrm{H}_{2} \mathrm{O} \underset{\mathrm{MeOH}}{\stackrel{\text { rt, air }}{\longrightarrow}} \mathrm{Na}_{2}\left[\mathrm{Ce}(\text { pic })_{5}\right] \cdot 4 \mathrm{H}_{2} \mathrm{O}
$$



$\alpha$-pyridoin

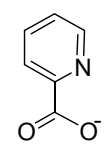

picolinate

(2-pyridinecarboxylate, pic)

Scheme 1 
Table 1. Crystal data and structure refinement for $\mathbf{1}, \mathrm{Na}_{2}\left[\mathrm{Ce}(\text { pic })_{5}\right]$. pic $\cdot 4 \mathrm{H}_{2} \mathrm{O}$

\begin{tabular}{ll}
\hline Chemical formula & $\mathrm{Na}_{2}\left[\mathrm{Ce}\left(\mathrm{C}_{6} \mathrm{H}_{4} \mathrm{NO}_{2}\right)_{5}\right]\left(\mathrm{C}_{6} \mathrm{H}_{5} \mathrm{NO}_{2}\right) 4\left(\mathrm{H}_{2} \mathrm{O}\right)$ \\
Formula weight & 991.78 \\
Temperature & $296(2) \mathrm{K}$ \\
Wavelength & $0.71073 \AA$ \\
Crystal system, space group & Monoclinic, $P_{2} / n$ \\
Unit cell dimensions & $a=12.8001(4) \AA$ \\
& $b=16.6933(6) \AA \quad \beta=93.093(2)^{\circ}$ \\
& $c=21.2088(6) \AA$ \\
Volume & $4525.2(3) \AA^{3}$ \\
$Z$, Calculated density & $4,1.456 \mathrm{Mg} / \mathrm{m}^{3}$ \\
$F(000)$ & 1996 \\
Crystal size & $0.18 \times 0.14 \times 0.13 \mathrm{~mm}$ \\
Reflections collected/unique & $28822 / 8009\left[R_{\text {int }}=0.0340\right]$ \\
Refinement method & $\mathrm{Full-matrix} \mathrm{least-squares} \mathrm{on} F^{2}$ \\
Data/restraints/parameters & $8009 / 7 / 578$ \\
Goodness-of-fit on $F^{2}$ & 1.077 \\
Final $R$ indices $[I>2 \sigma(I)]$ & $R_{1}=0.0327, w R_{2}=0.0869$ \\
$R$ indices (all data) & $R_{1}=0.0405, w R_{2}=0.0903$ \\
Largest diff. peak and hole & 1.370 and $-0.526 \mathrm{e} \AA^{-3}$ \\
\hline
\end{tabular}



Figure 1. ORTEP diagram of complex 1, $\mathrm{Na}_{2}\left[\mathrm{Ce}(\text { pic })_{5}\right] \cdot \mathrm{picH} \cdot 4 \mathrm{H}_{2} \mathrm{O}$, showing the atom numbering scheme with $30 \%$ probability ellipsoids. $\mathrm{H}$ atoms are omitted for clarity.

The $\mathrm{Ce}(\mathrm{III})$ ion is coordinated to ten $\mathrm{O}$ and $\mathrm{N}$ atoms in anionic $\left[\mathrm{Ce}(\mathrm{pic})_{5}\right]^{2-}$ complex which is shown in Figure 2(a). All five picolinates are coordinating cerium metal in N, O-chelating mode. The $\mathrm{Ce}-\mathrm{O}$ and $\mathrm{Ce}-\mathrm{N}$ bond distances are within the range of 2.465(2) 2.610(2) $\AA$ and 2.727(3) 2.777(3), respectively, and are slightly shorter or longer than the sum of the covalent radii of the $\mathrm{Ce}-\mathrm{O}(2.490 \AA)$ and $\mathrm{Ce}-\mathrm{N}(2.530 \AA)$. Selected bond lengths and angles are given in Table 2 . The other view of coordination polyhedron on Ce(III) ion is shown in Figure 2(b). The skeletal structure around the Ce atom forms a slightly distorted bicapped square antiprism. The $\mathrm{O} 8$ and $\mathrm{O} 26$ atoms, which are at relatively longer distances than the other $\mathrm{O}$ atoms, are on the bicapped positions. The bond angle of O8-Ce1-O26 is $171.9(1)^{\circ}$. And the other eight atoms (five $\mathrm{N}$ atoms and three $\mathrm{O}$ atoms) form a slightly distorted square antiprism.
Table 2. The selected bond distances $(\AA)$ and angles $\left(^{\circ}\right)$ of 1

\begin{tabular}{llll}
\hline Ce1-N1 & $2.776(3)$ & Ce1-O8 & $2.610(2)$ \\
Ce1-N10 & $2.777(3)$ & Ce1-O17 & $2.516(2)$ \\
Ce1-N19 & $2.767(3)$ & Ce1-O26 & $2.564(2)$ \\
Ce1-N28 & $2.735(3)$ & Ce1-O35 & $2.513(2)$ \\
Ce1-N37 & $2.727(3)$ & Ce1-O44 & $2.465(2)$ \\
Na1-O45 & $2.416(3)$ & Na2-O8 & $2.426(3)$ \\
Na1-O45 & $2.288(3)$ & Na2-O35 & $2.458(2)$ \\
Na1-N47 & $2.450(3)$ & Na2-O56 & $2.627(5)$ \\
Na1-O53 & $2.344(3)$ & Na2-O57 & $2.411(5)$ \\
Na1-O55 & $2.295(4)$ & Na2-O58 & $2.421(4)$ \\
Na1-O44 & $2.827(2)$ & Na2-O58 & $2.433(3)$ \\
O8-Ce1-N1 & $59.62(7)$ & O8-Ce1-N37 & $68.71(8)$ \\
O8-Ce1-O17 & $65.59(8)$ & O8-Ce1-O35 & $64.71(7)$ \\
O8-Ce1-O26 & $171.85(7)$ & N1-Ce1-O35 & $123.04(8)$ \\
O26-Ce1-N10 & $66.76(8)$ & O26-Ce1-O44 & $68.17(7)$ \\
O26-Ce1-N19 & $60.10(7)$ & O26-Ce1-N28 & $67.92(8)$ \\
N28-Ce1-O35 & $61.23(7)$ & N10-Ce1-O17 & $59.80(7)$ \\
N37-Ce1-O44 & $61.97(7)$ & & \\
\hline
\end{tabular}

Symmetry code: (i) $-\mathrm{x}+2,-\mathrm{y}+1,-\mathrm{z}+1$ (ii) $-\mathrm{x}+2,-\mathrm{y}+1,-\mathrm{z}+2$.

(a)

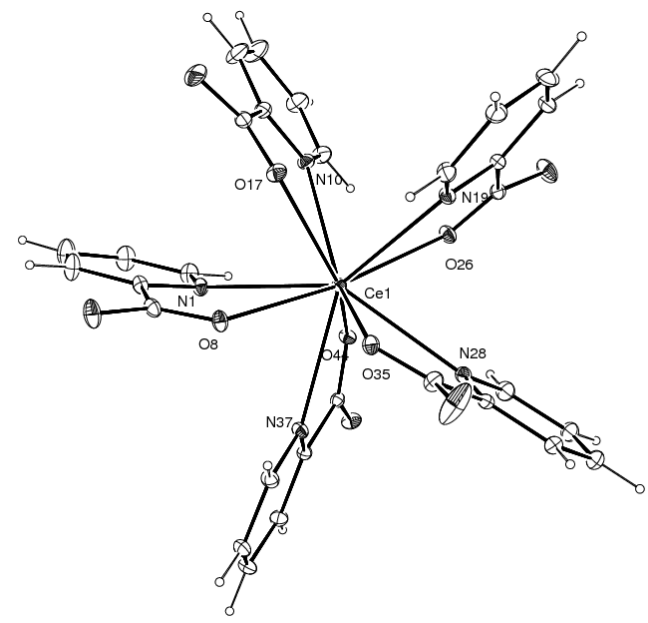

(b)



Figure 2. (a) ORTEP diagram of the anionic $\left[\mathrm{Ce}(\text { pic })_{5}\right]^{2-}$ in complex 1. (b) Distorted bicapped square antiprism of cerium(III) metal. Thermal ellipsoids of $30 \%$ are shown. 




Figure 3. Distorted octahedral geometry on $\mathrm{Na}$ atom (symmetry code: (i) $-\mathrm{x}+2,-\mathrm{y}+1,-\mathrm{z}+2)$.

Sodium ions are bounded with water molecules and with carboxylate oxygen atoms to form a distorted octahedral geometry, which was observed in $\mathrm{Na}\left[\mathrm{Ln}(\text { pic })_{4}\right](\mathrm{Ln}=\mathrm{Eu}$ (III) or $\mathrm{Tb}(\mathrm{III})$ ) already. ${ }^{6}$ As shown in Figure 3, Na atom is bounded with four water and two oxygen atoms of picolinate ligands.

The averaged bond distance of Ce(III)-oxygen of carboxylic group in complex $\mathbf{1}$ is $2.534 \AA$, which is consistent with the trends of reported data for lanthanide (III) picolinate complexes. The reported distances of metal-oxygen in $\mathrm{Nd},{ }^{7,9,10} \mathrm{Sm},{ }^{4,7,13}$ $\mathrm{Eu},{ }^{6} \mathrm{~Tb},{ }^{6} \mathrm{Dy},{ }^{8} \mathrm{Ho},{ }^{4,11} \mathrm{Er}^{12}$ complexes in a decreasing atomic radii are 2.460, 2.453, 2.390, 2.390, 2.342, 2.333 and 2.291 $\AA$, respectively. The averaged bond distance of cerium-nitrogen in pyridine ring is $2.756 \AA$, which is in the range of expected value. The reported distances of metal-nitrogen in $\mathrm{Nd},{ }^{7,9,10}$ $\mathrm{Sm},{ }^{4,713} \mathrm{Eu},{ }^{6} \mathrm{~Tb},{ }^{6} \mathrm{Dy},{ }^{8} \mathrm{Ho},{ }^{4,11} \mathrm{Er}^{12}$ complexes in a decreasing atomic radii are 2.674, 2.674, 2.619, 2.618, 2.520, 2.609, and $2.511 \AA$, respectively. The results conclude that the bond distances and coordination numbers are relevant with ion size. Therefore, ten-coordinated environment for cerium picolinate is predictable in comparison with nine or eight for other smaller lanthanide picolinates.

In conclusion, new cerium picolinate complex, $\mathrm{Na}_{2}\left[\mathrm{Ce}(\mathrm{pic})_{5}\right]$. picH $\cdot 4 \mathrm{H}_{2} \mathrm{O}$, has been prepared from the reaction of $\mathrm{CeCl}_{3} \cdot$ $7 \mathrm{H}_{2} \mathrm{O}$ and $\alpha$-pyridoin anion in methanol solution and structurally characterized as bicapped square antiprismatic geometry with ten coordination.

Acknowledgments. This work was supported by Dong-eui University Foundation Grant (2007AA115). X-ray data were collected at the Center for Research Facilities in Chungnam National University.

\section{References}

1. Armelao, L.; Quici, S.; Barigelletti, F.; Accorsi, G.; Bottaro, G.; Cavazzini, M.; Tondello, E. Coord. Chem. Rev. 2009.

2. Song, Y. S.; Yan, B.; Chen, Z. X. J. Mol. Struc. 2005, 750, 101.

3. Pack, Y. J.; Lee, H. H.; Kim, W. H.; Y. D. J. Coll. Inter. Science 1999, 209, 268.

4. Yun, S. S.; Kang, S. K.; Suh, H. R.; Suh, H. S.; Lee, E. K.; Kim, J. K.; Kim, C. H. Bull. Korean Chem. Soc. 2005, 26, 1197.

5. Zolin, V. F.; Tsaryuk, V. I.; Kudryashova, V. A.; Zhuravlev, K. P.; Gawryszewska, P.; Legendziewicz, J.; Szostak, R. J. Alloys. Compounds 2008, 451, 149.

6. Hong, J. H.; Oh, Y. H.; Kim, Y. M.; Kang, S. K.; Choi, J. W.; Kim, W. S.; Lee, J. I.; Kim, S. J.; Hur, N. H. Crystal Growth \& Design 2008, 8,1364 .

7. Li, W.; Wang, X. L.; Song, X. Y.; Li, L. C.; Liao, D. Z.; Jiang, Z. H. J. Mol. Struct. 2008, 885, 1.

8. Ma, J. F.; Hu, N. H.; Ni, J. Z. Polyhedron 1996, 15, 1797.

9. Starynowicz, P. Acata. Cryst. 1991, C47, 32.

10. Starynowicz, P. Acata. Cryst. 1991, C47, 294.

11. Starynowicz, P. Acata. Cryst. 1993, C49, 1895.

12. Soares-Santos, P. C. R.; Nogueira, HI. S.; Felix, V.; Drew, M. G. B.; Sa Ferreira, R. A.; Carlos, L. D.; Trindade, T. Inorg. Chem. Commun. 2003, 6, 1234.

13. Soares-Santos, P. C. R.; Almeida Paz, F. A.; Sa Ferreira, P. A.; Klinowski, J.; Carlos, L. D.; Trindade, T.; Nogueira, H. I. S. Polyhedron 2006, 25, 2471.

14. Sheldrick, G. M. Acta Cryst. 2008, A64, 112. 